\title{
Characterization of Transmission and Reflection of Ku-Band Split Ring Resonator Reflectarray using Waveguide Method
}

\author{
Noaman Naseer ${ }^{1}$, Birsen Saka ${ }^{1}$ \\ ${ }^{1}$ Department of Electrical and Electronics Engineering, Hacettepe University, Ankara, Turkey \\ *Noaman Naseer, E-mail: noaman. naseer@hacettepe.edu.tr
}

\begin{abstract}
The analysis, design, and measurement of a split ring resonator (SRR) reflectarray are presented. The 6 different designs of a $2 \times 4$ reflectarray are simulated to analyze the effect of split's positions on the resonance frequency. The SRR reflectarray which covers the highest frequency bandwidth at $\mathrm{Ku}$-band is fabricated and its transmission, reflection and absorbance coefficients are measured using the waveguide method. In the fabrication FR4 substrate is used. The S-parameter measurements of the fabricated reflectarray are also performed by the waveguide method. The results revealed good reflection characteristics for a wide frequency range from $12 \mathrm{GHz}$ to $16.5 \mathrm{GHz}$ in the $\mathrm{Ku}-$ band. The maximum value of reflection is obtained approximately at $15.3 \mathrm{GHz}$ frequency.
\end{abstract}

\section{Introduction}

In recent years the split ring resonators (SRR) played a vital role in radio frequency and microwave applications and their importance have increased with the advent of metamaterials. Many researchers also explored the application of SRR in reflectarray antennas. The X-band compact and high gain reflectarray unit cell using SRR is discussed in [1]. A parametric analysis of X-band Double Split Ring Resonator (DSRR) as reflectarray for linear polarization is presented in [2] whereas a frequency selective dual band $\mathrm{X} / \mathrm{Ku} \mathrm{SRR}$ reflectarray is studied in [3]. The resonance frequency of SRR is the most important factor which needs a detailed investigation of the effect of different geometrical parameters. An analytical and numerical study on the effect of the combination of SRR on its resonance frequency is presented in [4]. A study is presented in [5] to analyze the miniaturization performance of SRR loaded antenna due to the split ring arrangement and slit position. In [6], Moghbeli and co-authors investigated the response of electromagnetic properties of a single gap SRR unit cell by varying geometric parameters.

The SRRs due to their unique structure can develop absorbance ability in electromagnetic passive circuits [17]. For an efficient reflectarray design it is essential that a complete picture of transmission, reflection and absorbance should be known. The most common method used for transmission and reflection characterization of a material is the free space method. Effective properties of SRR from its scattering parameters are determined using free space method for $8 \mathrm{GHz}$ to $26 \mathrm{GHz}$ frequency band in [7]. However, the free space method is more suitable for reflectarrays having a large number of elements and measurements must be taken inside an expensive anechoic chamber. The alternative method more suitable for small size reflectarray antennas is the waveguide method. The characterization of the phase of the reflection coefficient of a hollow patch antenna at Ku-band using the waveguide method is successfully demonstrated by Hajian and coauthors [8] while reflection characteristics of reflectarray using S-band waveguide are explained in [8] and [9]. The Xband waveguide setup is used to find the reflection coefficient of Minkowski shaped microstrip reflectarray as discussed in [10]. The numerical and experimental validation of individual, two and four coupled element single ring SRRs having one, two and four gaps, the orientation of gaps with respected to incident wave is presented in [16].

The main drawback in microstrip reflectarrays is their narrowband performance which becomes more severe for small size reflectarrays. In this paper, we present computation and experimental validation of the effect of gap position and orientation of gap with respect to the incident wave and neighboring elements on the resonance frequency and bandwidth using the waveguide method. The three different orientations of SRR unit cell and six different orientations of SRR array are analyzed using the Computer Simulation Tool (CST). The design covering a wide frequency range in Ku-band is fabricated and its transmission and reflection characteristics are calculated from the S-parameters measured with the help of network analyzer.

\section{Unit cell design}

The basic structure of split ring resonator (SRR) consists of two split conducting rings having splits on opposite sides. When time harmonic electromagnetic fields are applied on SRR, being smaller in electrical size the field displacement lines easily transfer from one circle to the other through these splits which induce magnetic resonance. The SRR can be considered as an equivalent to the $\mathrm{LC}$ circuit $[11,12]$. The main geometrical parameters which affect the resonance frequency are the width of the conducting rings, the gap between the rings and split width of the rings. The gap 
between outer and inner rings and splits on them are considered as capacitive element whereas conducting rings are as inductive elements. When the external field is applied to the SRR, the current is induced along the ring which produces a magnetic moment. The induced current flows from one ring to the other through the slits present on both rings. The opposite charges accumulate on outer and inner rings and both sides of the slit. This is called distributed capacitance and gap capacitance respectively and SRR as a whole behave as an LC circuit. The unit SRR cell and its equivalent circuit are shown in Fig. 1.
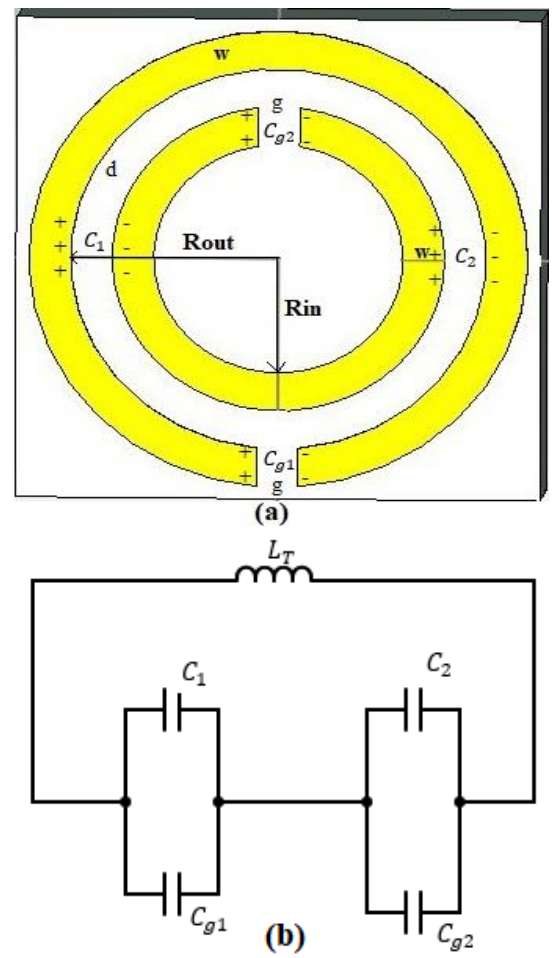

Figure 1: (a) Unit cell (b) Equivalent circuit.

The resonant frequency is calculated using the relation [12].

$$
\omega=\sqrt{\frac{2}{\left(C_{0}+C_{g}\right) L}}
$$

where $C_{0}$ is the mutual capacitance of the rings, $C_{\mathrm{g}}$ is the gap capacitance and $L$ is mutual inductance between the rings. If dimensions of both gaps $g$ are equal, then the gap capacitance $C g_{1}$ will be equal to the gap capacitance $\mathrm{Cg}_{2}$ and it can be replaced by the equivalent capacitance $C_{\mathrm{g}}$. Similarly, if the angle of rotation between the slit of outer and inner rings is $0^{\circ}$, distributed capacitance $C_{1}$ will be equal to $C_{2}$ and it can be replaced by $C_{0}$. When dimensions of gaps $g$ of outer and inner rings are different $C_{\mathrm{g} 1}$ will be different than $C_{\mathrm{g} 2}$. Similarly, if slit of one of the split ring is rotated with an angle the angular separation of slits varies leading to different values of $C_{1}$ and $C_{2}$. In this case, the revision of equation 1 is required to accommodate the effect of both capacitances. In this paper, we exploit the orientation of gap with respect to incident field and neighboring element to control the resonance frequency.

The numerical analyses of SRR reflectarray and unit cell are performed using Computer Simulation Tool CST. The substrate used in the simulation is FR4 having dielectric constant $\varepsilon_{\mathrm{r}}=4.3$ and thickness $t=1.4 \mathrm{~mm}$. The unit cell consists of two split rings without a back conducting plane, the radius of the inner ring is $1.2 \mathrm{~mm}$, the width of the conductor $w$ is $0.4 \mathrm{~mm}$, the distance $d$ between two rings is $0.4 \mathrm{~mm}$, the split $g$ is chosen as $0.4 \mathrm{~mm}$ wide, and the radius of the outer ring is $2 \mathrm{~mm}$. The width and length dimensions of the unit cell are $5 \mathrm{~mm}$ each. In the simulation, the SRR unit cell is placed between $\mathrm{Ku}$-band waveguide which is excited by the waveguide port having the dominant $\mathrm{TE}_{10}$ mode. The waveguide setup used in the simulation and the results of our calculations are shown in Fig. 2 and Fig. 3, respectively.

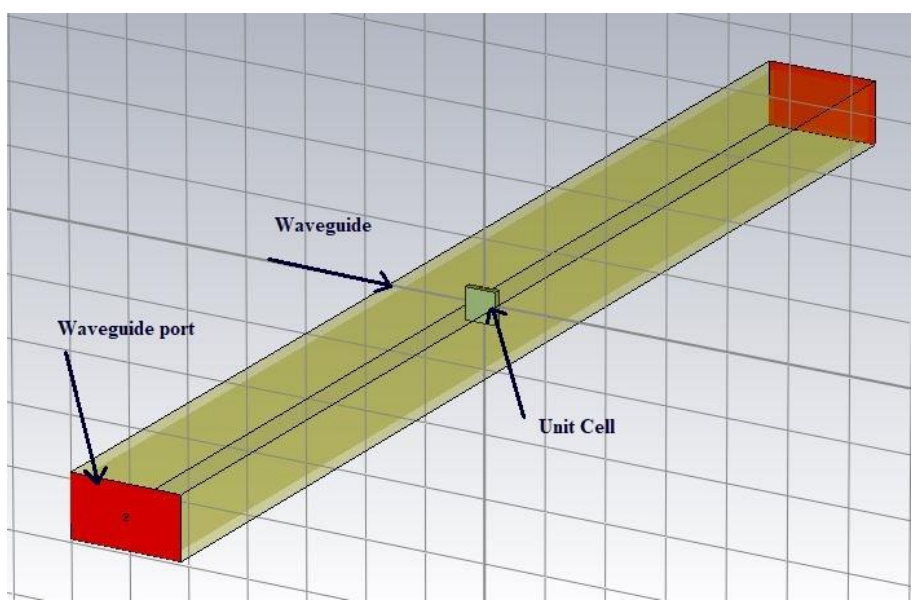

Figure 2: Simulated waveguide setup

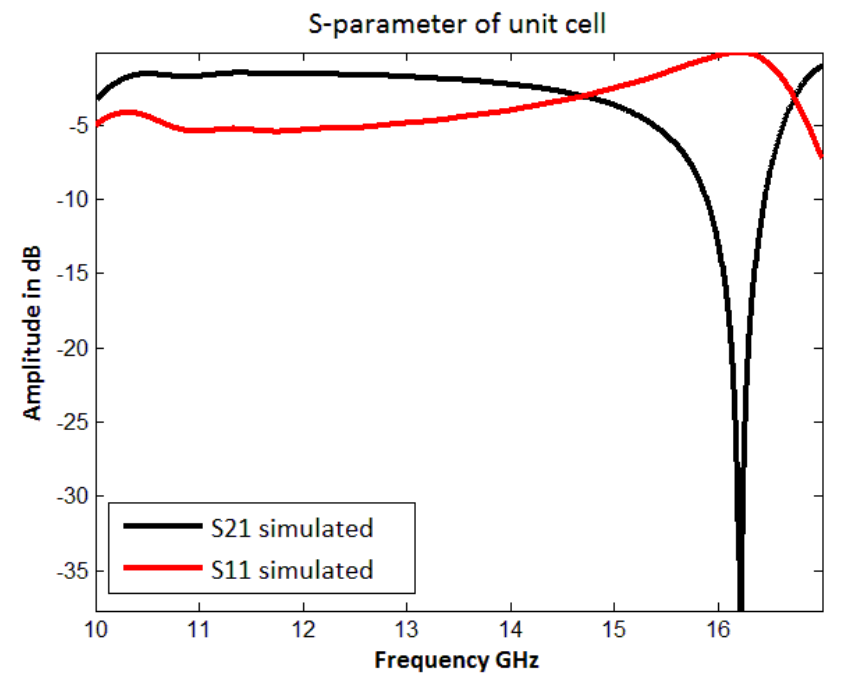

Figure 3: S-Parameters of the unit cell obtained through simulated waveguide setup.

It can be observed that the unit cell covers a small bandwidth from $15.9 \mathrm{GHz}$ to $16.42 \mathrm{GHz}$ and it has a maximum value of $\mathrm{S}_{11}$ near $16.2 \mathrm{GHz}$. 


\section{Reflectarray and measurement setup}

The six $2 \times 4$ SRR reflectarrays are simulated by varying the position of splits of conducting rings. In 4 designs splits are varied by the difference of $90^{\circ}$ along the axis while keeping the split of the inner ring opposite to the slit of the outer ring. In the $5^{\text {th }}$ design, the slit positions of outer and inner rings are varied from each other by $90^{\circ}$. The $6^{\text {th }}$ design is the optimized design in which the SRR of $1^{\text {st }}$ and $2^{\text {nd }}$ rows are the mirror images of each other as shown in Fig. 4.

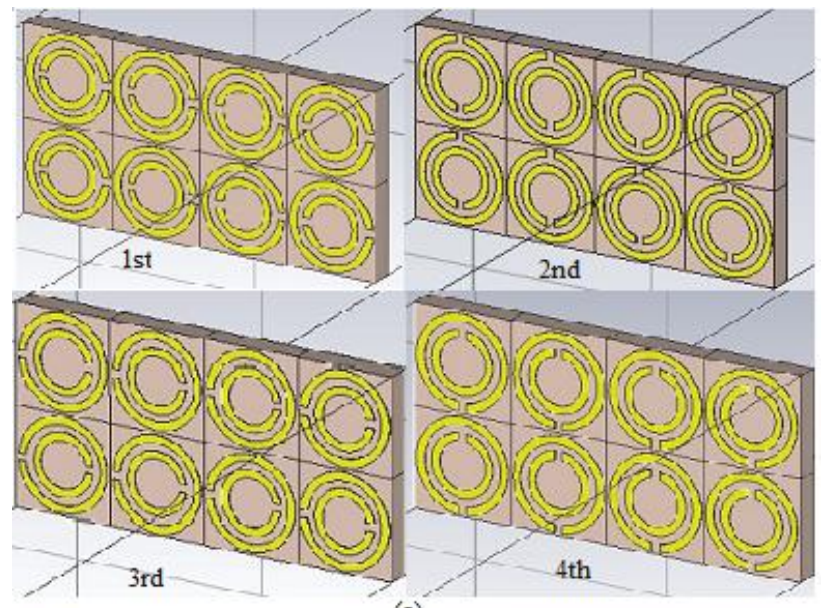

(a)

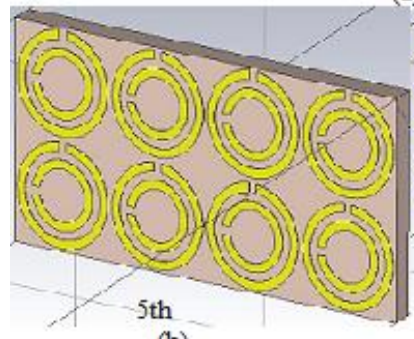

(b)

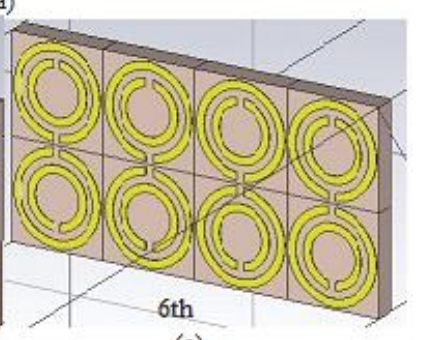

(c)
Figure 4: Simulated $2 \times 4$ SRR reflectarrays.

The simulation results of the reflectarrays are presented in Fig. 5 and Fig. 6.

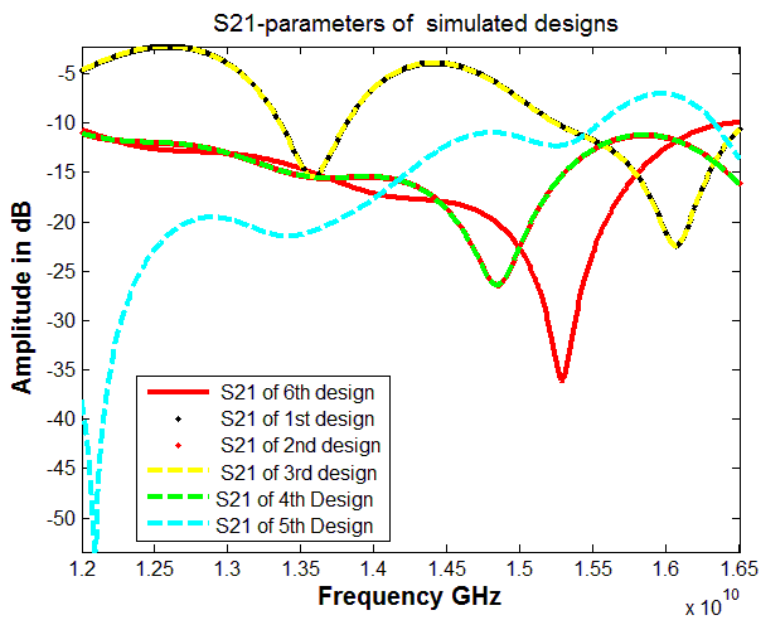

Figure 5: Effect of slit position on resonance frequency.

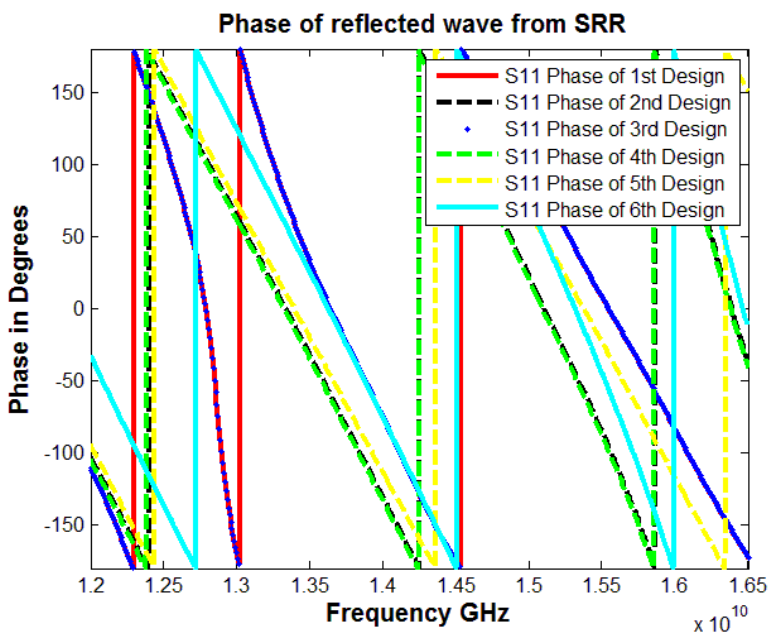

Figure 6: Simulation results of the phase of the reflected wave.

The resonance frequency of the unit cell as shown in Fig.3 is around $16.2 \mathrm{GHz}$ and it covers a very small bandwidth. The bandwidth maximization of SRR reflectarray is achieved by varying the slit position of SRR and keeping other geometrical parameters constant. In Fig. 5, the dotted black and yellow overlapping lines are $\mathrm{S}_{21}$ parameters of $1^{\text {st }}$ and $3^{\text {rd }}$ designs, the dotted red and green lines are for $2^{\text {nd }}$ and $4^{\text {th }}$ designs while dotted blue colored and solid red lines depict $5^{\text {th }}$ and $6^{\text {th }}$ designs, respectively. It is observed that as the slit position varies from the $1^{\text {st }}$ design to the $6^{\text {th }}$ the resonance frequency and bandwidth varies accordingly. For the $1^{\text {st }}$ and $3^{\text {rd }}$ designs the incident $\mathrm{E}$ field is perpendicular to the slit position and for $2^{\text {nd }}, 4^{\text {th }}$ and $5^{\text {th }}$ designs the incident field is parallel to slits. It is observed that when the slits are parallel to incident field the incident wave penetrates into the conducting rings through those slits and develops a strong coupling effect which results in an increase in overall capacitance and consequently, strong resonance is observed. In the $5^{\text {th }}$ design, the outer slit is parallel to the incident field but the inner slit is not. The angle of rotation of inner and outer slits is $90^{\circ}$ which make $C_{1}$ and $C_{2}$ unequal as a result the resonance frequency moving toward the end of the frequency band. In the $6^{\text {th }}$ design, the orientation produces a strong coupling effect to increase the bandwidth. It is found from the results in Figure 5 that the $6^{\text {th }}$ design covers the maximum bandwidth in Ku-band. Fig. 6 represents the phase of the reflected wave with respect to frequency for a normal incident which shows that phase of symmetric mirror designs has the same phase variation. Furthermore, it can be observed that the slit position also has an effect on the phase variation.

The optimized design, which covers a wide frequency band from $12 \mathrm{GHz}$ to $16.5 \mathrm{GHz}$, is fabricated and tested. The S-parameters of fabricated design are measured using the waveguide setup. The fabricated SRR reflectarray and the waveguide setup used in the measurement are shown in Fig. 7 (a) and (b) respectively. 


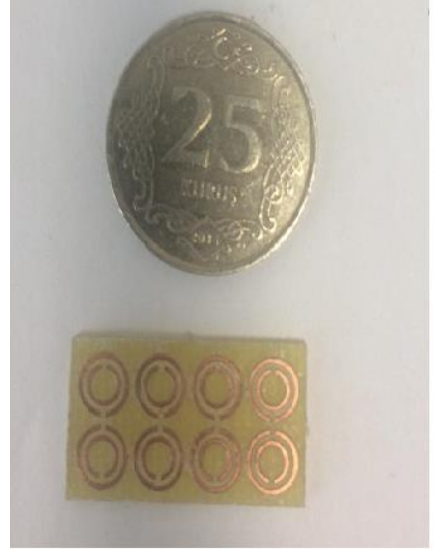

(a)

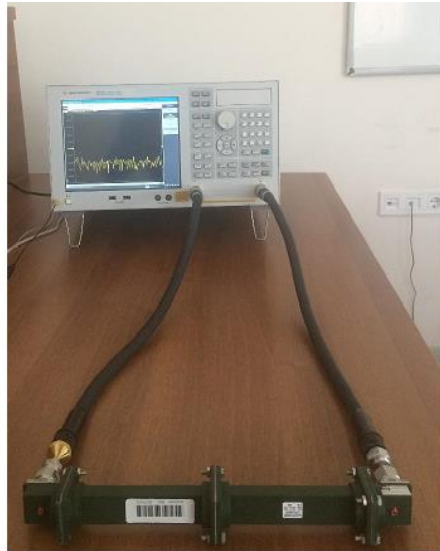

(b)
Figure 7: (a) 2x4 SRR reflectarray. (b) Waveguide setup.

The SRR reflectarray is placed between two air filled $\mathrm{Ku}-$ band rectangular waveguides. The opposite ends of the waveguides are connected to the network analyzer with the help of a coaxial cable to the waveguide adapters. The comparison of simulated and measured $S_{11}$ and $S_{21}$ parameters are shown in Fig. 8. The red and blue lines represent simulated and measured $\mathrm{S}_{11}$ while green and black lines show simulated and measured $S_{21}$, respectively. It can be observed evidently that both simulated and measured result are in good agreement and covering the bandwidth from $12 \mathrm{GHz}$ to $16.5 \mathrm{GHz}$ in the Ku-band very efficiently.

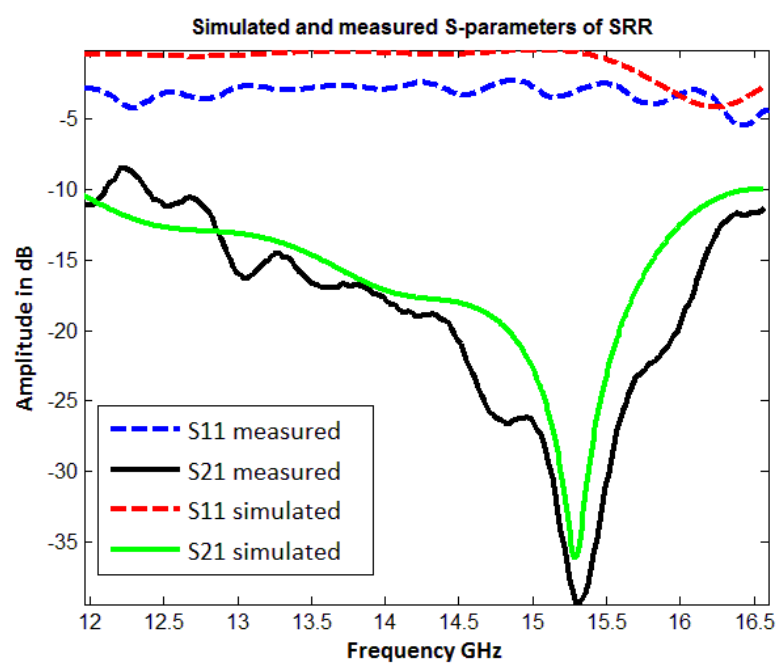

Figure 8: Simulated and measured results of $6^{\text {th }}$ design.

The phase of the simulated and measured result of the $6^{\text {th }}$ design is shown in Fig. 9. There is a phase difference of approximately $50^{\circ}$ between simulated and measured results due to instability of coaxial cables, poor sample preparation, sample thickness and the air gap. Another important aspect is connecting the measuring ports at the edges which require the transformation of the axis for exact measurements [10, $13]$.

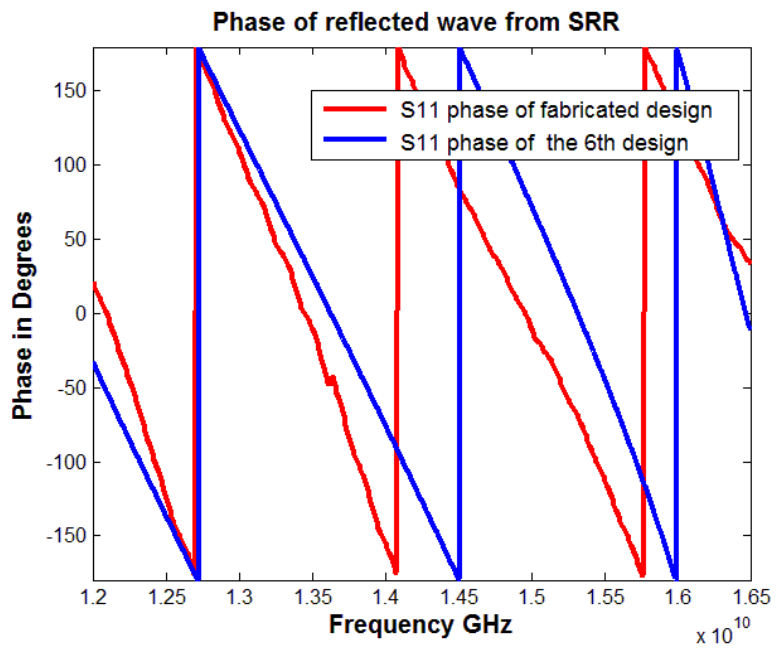

Figure 9: Simulated and measured results of the phase of the reflected wave.

The transmission, reflection and absorption coefficients are then calculated from measured S-parameters using the following equations [14].

$$
\begin{gathered}
T=\left|S_{12}\right|^{2} \\
R=\left|S_{11}\right|^{2} \\
A=1-T-R
\end{gathered}
$$

where $T, R$ and $A$ are the transmission, reflection and absorption coefficients, respectively. The calculated values using Eqs. 2, 3 and 4 are shown in Fig. 10.

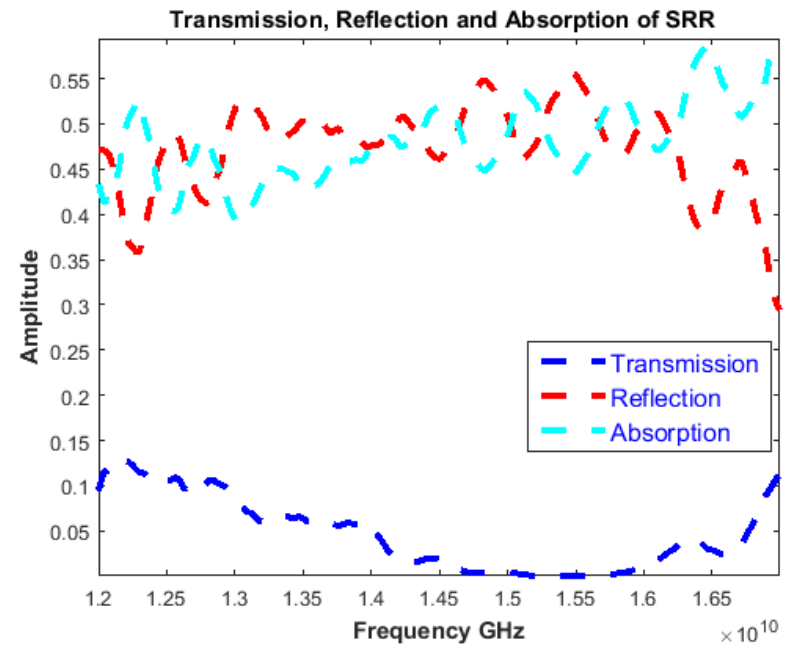

Figure 10: Transmission, Reflection and Absorption coefficients of fabricated reflectarray.

It can be seen from Fig.10 that most of the incident wave is either reflected back or absorbed in our design whereas a very small portion is transmitted. The transmission, absorption and reflection curves suggest that the proposed design have its application not only as a reflectarray but also as a frequency selective surface to reject the desired band [15]. 


\section{Discussion and Conclusion}

In this paper, we had two main objectives. The first one is to increase the bandwidth of a small SRR reflectarray. The second one is to determine the transmission and reflection characterization of a small reflectarray using the waveguide method. The six different designs are simulated to investigate the effect of slit orientation on bandwidth enhancement of SRR. We observed that the slit position of SRR has a significant influence on resonant frequency and bandwidth. The orientation of slit with respect to incident wave and neighboring element widens the bandwidth to cover maximum frequency band of interest. The simulated SRR reflectarray with optimum performance is fabricated and its $S_{11}$ and $S_{21}$ parameters are measured through the waveguide method. The measured S-parameters are in good agreement with simulation results.

\section{Acknowledgements}

We would like to thank Dr. Dinçer Gökcen for helping us in fabrication of the designs.

\section{References}

[1] Ahn, Chi-Hyung, Seong-Won Oh, and Kai Chang. "Compact reflectarray antenna element using split ring resonator." 2010 IEEE Antennas and Propagation Society International Symposium., IEEE, 2010.

[2] Özşahin, Gülay, et al. "Parametric analysis of double-split ring resonator as a reflectarray unit cell." 2017 IEEE International Symposium on Antennas and Propagation \& USNC/URSI National Radio Science Meeting. IEEE, 2017.

[3] Derafshi, Iman, et al. "Dual-band X/Ku reflectarray antenna using a novel FSS-backed unit-cell with quasi-spiral phase delay line." Journal of Microwaves, Optoelectronics and Electromagnetic Applications 15.3 (2016): 225-236.

[4] Radkovskaya, A., et al. "Resonant frequencies of a combination of split rings: Experimental, analytical and numerical study." Microwave and optical technology letters46.5 (2005): 473-476.

[5] Liu, Yanxia, Lotfollah Shafai, and Cyrus Shafai. "Effects of split ring arrangement and slit location on the miniaturization performance of split ring loaded dielectric resonator antennas." 2017 XXXIInd General Assembly and Scientific Symposium of the International Union of Radio Science (URSI GASS). IEEE, 2017.

[6] Moghbeli, Ebrahim, Hassan Ranjbar Askari, and Mohammad Reza Forouzeshfard. "The effect of geometric parameters of a single-gap SRR metamaterial on its electromagnetic properties as a unit cell of interior invisibility cloak in the microwave regime." Optics \& Laser Technology 108 (2018): 626-633.

[7] Varadan, Vasundara V., and Anilkumar R. Tellakula. "Effective properties of split-ring resonator metamaterials using measured scattering parameters: Effect of gap orientation." Journal of applied physics 100.3 (2006): 034910.

[8] Hajian, M., et al. "Design of hollow patch microstrip reflectarray and measuring phase of reflection coefficient at Ka-band using waveguide simulator." Proceedings of the Fourth European Conference on Antennas and Propagation. IEEE, 2010.

[9] Rajagopalan, Harish, and Yahya Rahmat-Samii. "On the reflection characteristics of a reflectarray element with low-loss and high-loss substrates." IEEE Antennas and Propagation Magazine 52.4 (2010): 73-89.

[10] Zubir, Farid, et al. "Design and analysis of microstrip reflectarray antenna with minkowski shape radiating element." Progress In Electromagnetics Research B 24 (2010): 317-331.

[11] Durán-Sindreu, Miguel, et al. "Electrically small resonators for planar metamaterial, microwave circuit and antenna design: A comparative analysis." Applied Sciences 2.2 (2012): 375-395.

[12] Li, B., et al. "A magnetic metamaterial-based spatial filter for phased-array applications." Materials Research Innovations 19.sup3 (2015): S70-S73.

[13] Tsipogiannis, Christos. "Microwave materials characterization using waveguides and coaxial probe." Mater's thesis, Lund University, (2012).

[14] Ahmad, Ahmad F., et al. "Attenuation Performance of Polymer Composites Incorporating NZF Filler for Electromagnetic Interference Shielding at Microwave Frequencies." J Material Sci Eng 5:289 (2016).

[15] Li, Long, et al. "Frequency selective reflectarray using crossed-dipole elements with square loops for wireless communication applications." IEEE Transactions on Antennas and Propagation 59.1 (2011): 89-99.

[16] Penciu, R. S., et al. "Multi-gap individual and coupled split-ring resonator structures." Optics Express 16.22 (2008): 18131-18144.

[17]Alici, Kamil Boratay, et al. "Experimental verification of metamaterial based subwavelength microwave absorbers." Journal of Applied Physics 108.8 (2010): 083113. 